\title{
HUMAN CYSTIC ECHINOCOCCOSIS: EVALUATION OF POST-TREATMENT SEROLOGIC FOLLOW-UP BY IgG SUBCLASS ANTIBODY DETECTION
}

\author{
STEPHEN D. LAWN, JOHN BLIGH, PHILIP S. CRAIG, AND PETER L. CHIODINI \\ Hospital for Tropical Diseases, London, United Kingdom; Cestode Zoonoses Research Group, School of Environment and Life \\ Sciences, University of Salford, Salford, United Kingdom; Department of Infectious and Tropical Diseases, London School of \\ Hygiene and Tropical Medicine, London, United Kingdom
}

\begin{abstract}
Assessment of post-treatment disease activity among patients with cystic echinococcosis (CE) is insensitive using detection of CE-specific total IgG antibody. This study investigated whether serum concentrations of CE-specific IgG subclasses 1-4 correlate better with disease activity than total IgG. We studied a cohort of patients $(\mathrm{n}=28)$ with symptomatic CE treated with anthelminthic drugs and surgery and who were followed up clinically and radiologically for a mean of 5.6 years (range $=3-12$ years). Serial archived sera collected during follow-up were retrospectively analyzed by enzyme-linked immunosorbent assays using crude horse hydatid cyst fluid as antigen. Changes in concentrations of antibodies were correlated with clinical and radiologic outcome. At diagnosis, concentrations of CE-specific total IgG, $\mathrm{IgG1}$, and IgG2 antibodies were significantly elevated in a greater proportion of patients compared with IgG3 and IgG4 antibodies. During post-treatment follow up, the $\mathrm{IgG} 2$ antibody response provided the best correlate of disease activity.
\end{abstract}

\section{INTRODUCTION}

Cystic echinococcosis (CE) is caused by infection with the larval stage (hydatid) of the cestode Echinococcus granulosus and is one of the world's major zoonotic infections. ${ }^{1}$ Humans acquire infection by accidental ingestion of E. granulosus eggs voided in the feces of infected dogs and the disease is common in parts of the world where there is close contact between the intermediate and definitive hosts, usually sheep and dogs, respectively. ${ }^{2}$ Cystic echinococcosis in humans usually presents with symptoms associated with the presence of fluid-filled cysts in the liver, lungs, or other viscera, and diagnosis is usually established by a combination of radiology and serology. ${ }^{3}$ Treatment modalities for CE include chemotherapy and surgery. ${ }^{2}$ Although surgery still remains the most common approach to treatment of CE throughout the world, long-term albendazole therapy has significant efficacy in approximately $70 \%$ of the cases. ${ }^{4}$ Albendazole is active against both cyst germinal membrane and protoscolices in animal models; ${ }^{5,6}$ praziquantel is protoscolicidal and may act synergistically with albendazole. ${ }^{7,8}$ However, the optimum duration and dosages of these drugs and their use in relation to surgical intervention have not been definitively determined. This is partly because $\mathrm{CE}$ is a chronic disease in which changes in disease activity and treatment effect are difficult to measure. As a result, no standardized approach to treatment has emerged.

Longitudinal assessments of hydatid cyst viability are commonly made using serial radiology with or without serial serology. However, there is no test for cure and follow-up surveillance is therefore required for many years. At the Hospital for Tropical Diseases (HTD) in London, an enzymelinked immunosorbent assay (ELISA) measuring CE-specific total $\mathrm{IgG}$ is the main serologic test used for diagnosis and follow-up. However, our experience with this test is that although there may be a gradual decrease in optical density (OD) over time following treatment, the test result rarely becomes negative even after many years, even in those whose disease is apparently cured. Similarly, serial radiology is often unreliable in establishing parasitologic cure; cysts killed by chemotherapy may persist and post-operative defects cannot always be distinguished from viable cysts.

Previous studies have reported that IgG subclasses 1-4 are differentially expressed in patients with chronic helminthic infections. ${ }^{9-11}$ Similarly, cross-sectional studies of patients with CE using an ELISA incorporating partially purified hydatid cyst fluid antigen B have found a predominance of IgG1 and IgG4 expression in serum. ${ }^{12-15}$ Moreover, serum concentrations of IgG4 are greater in patients with symptomatic CE compared with those with asymptomatic disease. ${ }^{15}$ It has therefore been suggested that IgG subclass measurements may provide a more sensitive index of disease activity than CE-specific total IgG.

Patients at HTD with symptomatic CE are treated and followed-up for many years with detailed longitudinal monitoring of clinical, radiologic, and serologic parameters. In this study, we aimed to conduct a retrospective longitudinal analysis of $\mathrm{IgG}$ subclass expression in patients with treated $\mathrm{CE}$ to determine whether serum concentrations of these subclass antibodies provide a better correlate of CE activity compared with CE-specific total IgG.

\section{PATIENTS AND METHODS}

Patients. A retrospective study of patients with CE was conducted at HTD after approval from the University College London Hospitals Committee on the Ethics for Human Research. A clinical database cross-checked with laboratory records was used to identify patients with diagnoses of symptomatic CE between 1990 and 2000. Serial serum samples obtained from these patients at the time of diagnosis and during out-patient follow-up had been stored at $-20^{\circ} \mathrm{C}$. Serologic and radiologic assessments of clinically quiescent $\mathrm{CE}$ were made at least annually and often more frequently. $\mathrm{Pa}-$ tients were included in this study if the case definition for CE was fulfilled and if sera had been stored at the time of initial diagnosis of active disease and at two or more follow-up time points. There was no selection of cases based upon previous serologic information. Using patient clinical records, we recorded the following data: demographic details, anatomic locations of cysts, clinical complications, results of radiologic investigations, details of surgical procedures, anthelminthic treatment, and histopathology of surgically resected specimens. The clinical and radiologic follow-up records of each patient were reviewed by two investigators (SDL and PLC). Before the serologic results of this study were known, patients 
were categorized as having cured or inactive disease and episodes of relapse were also identified. Archived sera from patients who had been investigated for possible CE but who had been found to have a different pathology were used as negative controls.

Treatment. The combined medical and surgical approach to treatment of $\mathrm{CE}$ at this institution has been previously reported. ${ }^{16}$ Where possible, patients receive albendazole, 400 mg twice a day for $\geq 3$ months, combined with praziquantel, $40 \mathrm{mg} / \mathrm{kg} /$ day for two weeks prior to surgical intervention. A minority of patients in this series were treated by medical means alone, using repeated courses of albendazole in combination with a single course of praziquantel. The most common surgical procedure performed was cystectomy using a freezing cone to prevent intra-operative spillage of cyst contents. Two patients were treated surgically by the puncture, aspiration, injection, reaspiration (PAIR) procedure. ${ }^{17}$

Definitions. Cases. Cases of $\mathrm{CE}$ were defined as patients with 1) histologically confirmed $\mathrm{CE}$ or 2) radiologic imaging results consistent with $\mathrm{CE}$ and positive serology.

Cure. A patient with $\mathrm{CE}$ was defined as having cured disease if the following three criteria were fulfilled: 1) complete surgical resection of all cyst material without spillage of cyst contents; 2) treatment with albendazole, $400 \mathrm{mg}$ twice a day for $\geq 3$ months plus praziquantel, $40 \mathrm{mg} / \mathrm{kg} /$ day for two weeks; and 3) no clinical or radiologic evidence of recurrence during $\geq 3$ years of follow-up.

Inactive. A patient with $\mathrm{CE}$ was defined as having inactive disease if the following three criteria were fulfilled: 1) surgical resection was not performed, was incomplete, or was associated with intra-operative spillage; 2) anthelminthic treatment with albendazole, $400 \mathrm{mg}$ twice a day for $\geq 3$ months plus praziquantel, $40 \mathrm{mg} / \mathrm{kg} /$ day for two weeks; and 3) there was no clinical or radiologic evidence of cyst activity or recurrence during $\geq 3$ years of follow-up.

Relapse. Relapsed CE was defined as a recurrence of active CE following a period of quiescent disease as shown by the radiologic enlargement of existing cysts and/or the development of new daughter cysts.

Serology. At HTD, an ELISA incorporating crude $E$. granulosus horse hydatid cyst fluid antigen is the main serologic test routinely used to measure total CE-specific IgG antibody. Using cyst material from a horse rather than from a human overcomes the problem of reduced ELISA specificity due to contamination with human antigens. This assay has a sensitivity of approximately $90 \%$ and a specificity of approximately $96 \%$ in detecting hepatic CE among the selected patient population at HTD (Chiodini PL, unpublished data). Suspected cases with borderline positivity may be further tested with an immunoblot. Complement fixation tests were not performed in this study because our own experience is that this test lacks sensitivity and shows significant inter-test variability. The ELISAs were all optimized by checkerboard titration using defined positive and negative sera and standard laboratory methods. Samples from the same patient were analyzed concurrently. The OD cut-off for each of the five assays was determined as the mean $+3 \mathrm{SD}$ of the negative control sera $(\mathrm{n}=20)$.

In these assays, $200-\mu \mathrm{L}$ volumes were used throughout unless otherwise stated. Immunolon-II high-binding microtiter plates (Dynatech, Chantilly, VA) were coated overnight at room temperature with centrifuged crude hydatid cyst fluid ( 9 $\mu \mathrm{g} / \mathrm{mL})$. Plates were washed four times with phosphatebuffered saline, $\mathrm{pH} 7.2,0.05 \%$ Tween (PBS-Tween) and the wells were then blocked with $3 \%$ non-fat milk in PBS-Tween for 60 minutes at room temperature. For the assays for total $\mathrm{IgG}, \mathrm{IgG} 1$, IgG2, IgG3, and IgG4, patient sera were diluted in PBS-Tween 1:500, 1:200, 1:100, 1:50, and 1:50, respectively. Diluted sera were incubated in duplicate wells of the microtiter plate for 90 minutes at $30^{\circ} \mathrm{C}$ and the plates were then washed four times with PBS-Tween. The different assays were then continued as follows.

Total IgG assay. Rabbit anti-human horseradish peroxidase-conjugated IgG (Dako, Ely, Cambridgeshire, United Kingdom) diluted 1:4,000 in PBS-Tween was added to each well and the plate was incubated for 90 minutes at $30^{\circ} \mathrm{C}$. The plate was then washed four times with PBS-Tween. A substrate solution of $4 \mathrm{mg} / \mathrm{ml}$ of $o$-phenylenediamine dihydrochloride (Sigma, St. Louis, MO) plus $0.005 \%$ hydrogen peroxide was added and incubated at room temperature for 15-20 minutes. Color development was stopped with $2.5 \mathrm{M}$ sulfuric acid $(30 \mu \mathrm{L})$ and absorbance values (ODs) were measured at $490 \mathrm{~nm}$ with an ELISA plate reader.

IgG subclass assays. For the IgG subclass assays, monoclonal mouse anti-human IgG1, IgG2, IgG3, and IgG4 (Sigma) diluted 1:500, 1:2,500, 1:1,000 and 1:2,500, respectively, was added to each well. The plates were then incubated for 90 minutes at $30^{\circ} \mathrm{C}$ and washed four times with PBS-Tween. Peroxidase-conjugated goat anti-mouse Fc-specific IgG (Sigma) was used as the secondary antibody and was diluted 1:24,000, 1:10,000, 1:4,000, and 1:24,000 in PBS-Tween for the $\mathrm{IgG} 1, \mathrm{IgG} 2, \mathrm{IgG} 3$, and IgG4 assays, respectively. Plates were incubated for 60 minutes at $30^{\circ} \mathrm{C}$ and the microtiter plate was then washed four times with PBS-Tween. Substrate solution was then added and the plate was developed and read as described for the total $\mathrm{IgG}$ assay.

Western blots. Crude E. granulosus antigen from horse hydatid cyst fluid was separated alongside molecular weight markers using polyacrylamide gel electrophoresis according to standard procedures. ${ }^{18}$ The $12 \%$ acrylamide resolving gel was overlaid with a $5 \%$ stacking gel. The separated antigens were transferred onto a nitrocellulose (NC) membrane (Hybond-P; Amersham Pharmacia Biotech, Little Chalfont, Bucks, United Kingdom) using a Semi-Phor semi-dry transfer unit (Hoefer Scientific Instruments, San Francisco, CA). Before immunodetection, the $\mathrm{NC}$ membrane was blocked with $3 \%$ non-fat milk in $50 \mathrm{mM}$ PBS (Blotto). The NC membrane was washed three times with $50 \mathrm{mM}$ PBS, $0.05 \%$ Tween, cut into strips approximately $4 \mathrm{~mm}$ wide, and placed in a multichannel plastic tray. One milliliter of a diluted serum (1:50 in blotto) was applied to each NC strip and incubated for one hour at room temperature. The blots were then washed three times with PBS, $0.05 \%$ Tween, and goat anti-human IgG conjugated with horseradish peroxidase diluted 1:500 in blotto was added to each NC strip and incubated for one hour at room temperature. The strips were washed again. Color development of the bands was achieved by incubating each NC strip for 30 minutes with chloronaphthol (3\% 4-chloro-1naphthol in absolute alcohol) diluted 1:100 in $50 \mathrm{mM}$ Tris$\mathrm{HCl}, \mathrm{pH} 7.6,0.03 \%$ hydrogen peroxide. The reaction was terminated by dilution of the substrate in a large volume of distilled water.

Data analysis. An OD at diagnosis $\geq 0.25$ was taken as the cut-off above which an assay might potentially provide useful 


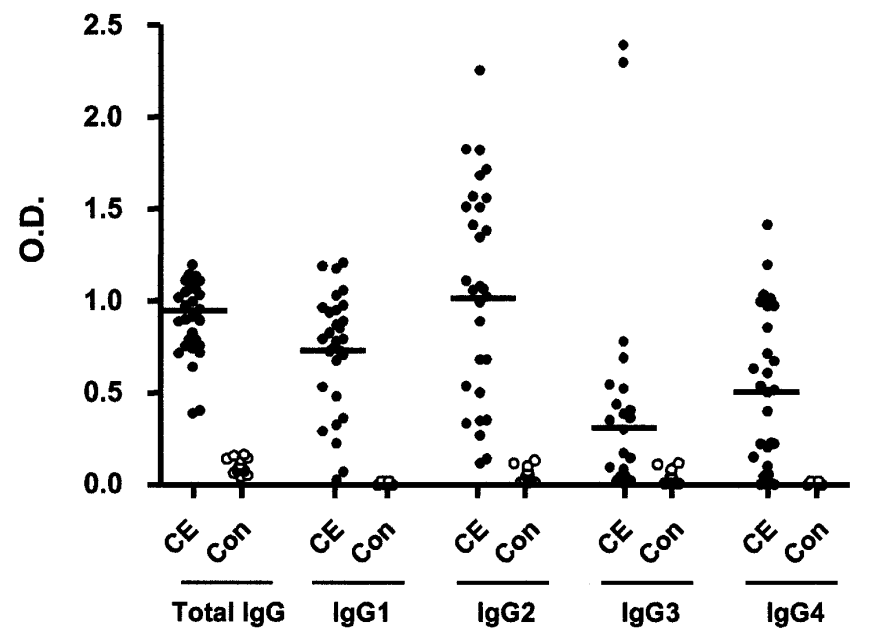

FIGURE 1. Total IgG, IgG1, IgG2, IgG3, and IgG4 antibody levels measured by an enzyme-linked immunosorbent assay in serum samples from patients with active symptomatic cystic echinococcosis (CE) $(\mathrm{n}=28)$ and controls (Con) who did not have CE $(\mathrm{n}=20)$. The graph shows the optical density (O.D.) values for each sample analyzed. Mean OD values in the $\mathrm{CE}$ groups are indicated by the horizontal bars.

information for post-treatment follow-up. The ELISA ODs increased in a proportion of the patients in the three months following surgical treatment of CE cysts, presumably because of antigen release. Depending on which was the greater, either the OD at diagnosis or the post-operative ( $<3$ months) OD was selected as the baseline value for post-treatment serologic follow-up. The proportion of patients with a change in OD $(\Delta \mathrm{OD}) \geq 0.2$ and $\geq 0.5$ during follow up were determined for each assay. Among patients with cured or inactive disease, group comparisons were made between peak ODs and ODs of the latest follow-up serum samples. Since the ODs were normally distributed, parametric statistical tests were used for group comparisons. Prism version 2.0 software (GraphPad Software Inc., San Diego, CA) was used for statistical analysis.

\section{RESULTS}

Cases of CE. Twenty eight patients (14 males and 14 females) fulfilled the inclusion criteria. Their mean \pm SD age was $37.1 \pm 15.6$ years and all but one were immigrants to the United Kingdom from countries in the Mediterranean, Balkans, or the Middle East. Cysts were confined to the abdomen in 19 patients (hepatic only $=14$, splenic only $=2$, multiple organs $=3$ ), were intrathoracic in three patients, and were present in both the thorax and abdomen in four patients; pelvic disease and spinal disease each occurred in one patient. Cysts were single in 13 patients and multiple in 15 patients. All 28 patients received anthelminthic treatment (minimum treatment of albendazole, $400 \mathrm{mg}$ twice a day for $\geq 3$ months, plus praziquantel, $40 \mathrm{mg} / \mathrm{kg} /$ day for two weeks). In addition, 25 of the patients underwent surgical procedures. The mean \pm SD duration of follow-up after diagnosis was $5.5 \pm 2.6$ years (range $=3-12$ years). Fifteen patients fulfilled the case definition for cure' and 11 for inactive disease. Relapse occurred in two patients. The mean number of serial serum samples analyzed per patient was 8.1 (range $=3-34$ ).

Serology at diagnosis. Cut-off ODs (mean +3 SD) derived from analysis of negative control sera were low for each of the assays: total IgG (0.217), IgG1 (0.028), IgG2 (0.168), IgG3 (0.163), and IgG4 (0.009) (Figure 1). Among the cases of CE studied, the number with positive serology ( $\geq$ cut-off level) at diagnosis was 28 of 28 (total $\mathrm{IgG}$ ), 28 of 28 (IgG1), 26 of 28 (IgG2), 13 of 28 (IgG3), and 23 of 28 (IgG4). Thus, the total $\mathrm{IgG}, \mathrm{IgG} 1$, and IgG2 assays had greater diagnostic sensitivity than the IgG3 and IgG4 assays. Similarly, the mean \pm SD ODs obtained with the total $\mathrm{IgG}, \mathrm{IgG} 1$, and IgG2 ELISAs $(0.956 \pm 0.204,0.805 \pm 0.326$, and $1.216 \pm 0.539$, respectively $)$ were significantly greater than those of the IgG3 and IgG4 assays $(0.389 \pm 0.608$ and $0.524 \pm 0.395$, respectively) (Figure 1$)$.

The proportions of patients at diagnosis with an OD $\geq 0.25$ obtained with the total $\mathrm{IgG}, \mathrm{IgG} 1$, and $\mathrm{IgG} 2$ ELISAs were 28 of 28,25 of 28 and 26 of 28, respectively, compared with 12 of 28 and 17 of 28 with the IgG3 and IgG4 ELISAs, respectively. These results indicated that measurement of IgG3 and IgG4 subclasses would not be useful for post-treatment follow-up of a significant proportion of patients.

Serology in patients with cured CE. Patients with cured disease $(n=15)$ were followed-up for a mean \pm SD of $4.9 \pm$ 1.8 years. The mean OD obtained with each of the five ELISAs decreased substantially between the peak values during active disease and values when cured (Table 1). The greatest mean decrease in OD $(\Delta \mathrm{OD})$ was observed with the $\mathrm{IgG} 2$ assay and this was significantly greater than the mean $\Delta \mathrm{OD}$ with the total $\mathrm{IgG}$ assay.

A $\Delta \mathrm{OD} \geq 0.2$ was seen in the great majority of patients with the total IgG, IgG1, and IgG2 assays, but in comparatively few with the IgG3 and IgG4 assays (Table 1). When the total $\mathrm{IgG}, \mathrm{IgG} 1$, and IgG2 assays were compared, the proportion of patients with a $\Delta \mathrm{OD} \geq 0.5$ was much smaller with the total IgG assay. In view of this observation, serial longitudinal changes in $\mathrm{IgG} 1, \mathrm{IgG} 2$, and total $\mathrm{IgG}$ were plotted for the

TABLE 1

Mean \pm SD ELISA OD values of total Echinococcus-specific $\mathrm{IgG}$ and $\mathrm{IgG}$ subclass antibodies in patients with cystic echinococcosis $(\mathrm{n}=15)$ at diagnosis and following cure*

\begin{tabular}{lcccrr}
\hline & Peak OD & Cured OD & $\Delta$ OD $\dagger$ & No. $\Delta$ OD $\geq 0.2$ & No. $\Delta$ OD $\geq 0.5$ \\
\hline Total IgG & $0.974 \pm 0.134$ & $0.442 \pm 0.213$ & $0.532 \pm 0.166$ & 15 & 7 \\
IgG1 & $0.886 \pm 0.254$ & $0.265 \pm 0.213$ & $0.638 \pm 0.215$ & 15 & 11 \\
IgG2 & $1.123 \pm 0.564$ & $0.354 \pm 0.201$ & $0.772 \pm 0.453$ & 13 & 12 \\
IgG3 & $0.211 \pm 0.212$ & $0.059 \pm 0.067$ & $0.148 \pm 0.172$ & 5 & 1 \\
IgG4 & $0.449 \pm 0.412$ & $0.080 \pm 0.412$ & $0.418 \pm 0.34$ & 8 & 7 \\
\hline
\end{tabular}

* The mean post-treatment decrease in OD $(\Delta \mathrm{OD})$ and the number of patients with a post-treatment decrease in OD $\geq 0.2$ (No. $\Delta$ OD $\geq 0.2)$ and $\geq 0.5$ (No. $\Delta$ OD $\geq 0.5)$ are given. ELISA

$\dagger$ By $t$-test. Total IgG vs IgG1 $(P=0.110)$. Total IgG vs IgG2 $(P=0.041)$. 
patients $(\mathrm{n}=8)$ in whom the convalescent total IgG OD remained $>0.5$ despite cure (Figure 2 ). In the majority of these cases, the dynamic range of the $\mathrm{IgG} 2$ ODs was greater than that of the total $\mathrm{IgG}$ and $\mathrm{IgG} 1$ ELISAs. Furthermore, in some of the patients (e.g., H1, H4, and H12), ODs for the total $\mathrm{IgG}$ and IgG1 ELISAs remained persistently elevated post-treatment, whereas a much greater reduction was seen in ODs obtained with the IgG2 assay.

Serology in patients with inactive CE. The patients with inactive $\mathrm{CE}(\mathrm{n}=11)$ were followed-up for a mean $\pm \mathrm{SD}$ of 5.5 \pm 2.6 years. The mean OD obtained with each of the five different ELISAs decreased following treatment (Table 2); decreases from the peak value were statistically significant for all assays except for the IgG3 ELISA. The greatest decrease in mean OD was seen with the IgG2 ELISA and a decrease $\geq$ 0.2 or $\geq 0.5$ occurred in a greater proportion of patients tested with this assay compared with the others (Table 2). The mean $\Delta$ ODs in the inactive group were far smaller than those in the cured group with the IgG1 $(P<0.001)$ and the total IgG $(P<$ $0.001)$ assays. In contrast, the decrease in OD with the IgG2 assay was of similar magnitude when the cured and inactive groups were compared $(P=0.130)$.

Serology and relapsed CE. Relapse of CE occurred in two patients during follow-up (Figure 3). Relapse of disease in patient $\mathrm{H} 2$ was accompanied by a substantial increase in $\mathrm{CE}$ specific total IgG, IgG1, and IgG2. However, relapse of disease in patient $\mathrm{H} 31$ was accompanied by a marked increase in OD only with the IgG2 ELISA; the ODs of the total IgG and IgG1 remained $<0.4$.

Western blots. Having demonstrated that the IgG2 antibody response appeared to correlate better with $\mathrm{CE}$ activity compared with the other subclasses, we made an attempt to determine the predominant parasite antigen recognized by IgG2 subclass antibodies. Eight serum samples were selected from patient $\mathrm{H} 2$ at time points when the patient's disease was diagnosed, post-treatment, during active relapse, and when disease was again inactive (Figure 3 ). Western blots to detect $\mathrm{CE}$-specific total IgG in the serum at the time of diagnosis of CE showed multiple bands of approximately 110-120, 38, 24, 16 , and $8 \mathrm{kD}$. In contrast, the blots to detect $\mathrm{IgG} 2$ showed a dominant $8-\mathrm{kD}$ band and weak bands of 38 and $100-120 \mathrm{kD}$. Following treatment, these bands in the $\operatorname{IgG} 2$ blots were barely visible but reappeared with a dominant $8-\mathrm{kD}$ band during the patient's disease relapse. Thus, we demonstrated for patient $\mathrm{H} 2$ that $\mathrm{CE}$-specific $\mathrm{IgG} 2$ antibodies predominantly bound to the 8-kD subunit of E. granulosus antigen B at diagnosis and during disease relapse.

\section{DISCUSSION}

This study provides a longitudinal assessment of changes in Echinococcus-specific total $\mathrm{IgG}$ and $\mathrm{IgG}$ subclass antibody responses in patients with $\mathrm{CE}$ receiving treatment for symptomatic disease. The detailed clinical, radiologic, and serologic follow-up of these patients longitudinally over a 3-12year period provided the opportunity to correlate $\mathrm{IgG}$ subclass antibody responses with treatment outcome, whereas previous studies have been cross-sectional in design. ${ }^{12-15,19}$ We found that at diagnosis the ELISA OD was significantly elevated in a greater proportion of patients with the total $\mathrm{IgG}$, $\mathrm{IgG} 1$, and $\mathrm{IgG} 2$ assays compared with the $\mathrm{IgG} 3$ and $\mathrm{IgG} 4$ assays. Importantly, serum concentrations of CE-specific IgG2 correlated most strongly with clinical outcome.

In the present study, an ELISA was used that incorporated crude E. granulosus hydatid cyst fluid rather than purified antigen (antigen B or antigen 5) as used in previous studies. $^{12,13,15,19}$ Our rationale was that the broad diversity of antigens present in crude hydatid cyst fluid might enhance detection of subclass antibodies with different antigen specificities. Despite using crude hydatid cyst fluid in the ELISAs, background OD values among the negative controls were low. Furthermore, a wide range of ODs was observed in all four assays used and were in general higher than those generated in assays incorporating purified antigen B. Although some previous studies ${ }^{12-15}$ found a predominance of IgG1 and/or IgG4 antibody responses in patients with symptomatic $\mathrm{CE}$ at diagnosis, our data agree with those of Ramzy and others, who using crude hydatid cyst fluid as antigen, found that ELISAs for total $\mathrm{IgG}, \mathrm{IgG} 1$, and $\mathrm{IgG} 2$ antibodies were the most sensitive. ${ }^{20}$ In the present study, low IgG3- and IgG4-specific antibody levels in a significant proportion of patients with $\mathrm{CE}$ at diagnosis effectively excluded these antibody subclasses as useful serologic markers for posttreatment follow-up.

Although there is no definitive test of cure among patients treated for symptomatic advanced CE, we believe that our case definition provided a very high likelihood of cure. These data confirm our previous clinical experience that detection of CE-specific total IgG antibody levels, while providing the basis for a sensitive diagnostic test, are unhelpful in serologic follow-up in a significant proportion of patients with CE. In contrast, in the present study, the broad dynamic range of serum $\operatorname{IgG} 2$ antibody concentrations and the correlation with treatment response and with relapse suggest that serum CEspecific IgG2 antibody concentrations may provide a useful index of post-treatment disease activity.

Eleven of the patients with $\mathrm{CE}$ were classified as having inactive disease because they were treated by medical means alone or because it was not possible to remove all cysts in some patients with extensive disease. However, all received chemotherapy and showed a clinical and radiologic response to treatment without relapse over many years of follow-up. It is likely that many of these patients were actually cured of their disease. The presence of residual dead cyst material may have provided an ongoing antigenic stimulus to antibody production and, in keeping with this, the post-treatment changes in antibody levels ( $\Delta$ ODs) for patients classified as having inactive disease were in general smaller than those with cured disease (Tables 1 and 2). The notable exception to this was the IgG2 assay, which showed similar post-treatment $\Delta \mathrm{ODs}$ in both cured and inactive disease groups. It is possible that IgG2 concentrations may correlate more closely with cyst viability and may be independent of the presence of persistent dead cyst material. More prolonged follow-up of this cohort of patients will establish whether indeed this is the case.

In clinical practice, early detection of relapse of CE may be difficult. In patient $\mathrm{H} 2$, a marked increase in concentrations of total $\mathrm{IgG}, \mathrm{IgG} 1$, and $\mathrm{IgG} 2$ antibodies all correlated with disease relapse (Figure 3 ). In contrast, it was striking that specific IgG2 was the only serologic correlate of disease relapse in patient H31 (Figure 3). Had IgG subclass measurements been made in this patient during follow-up, the increasing concentration of $\mathrm{IgG} 2$ over several years might have 

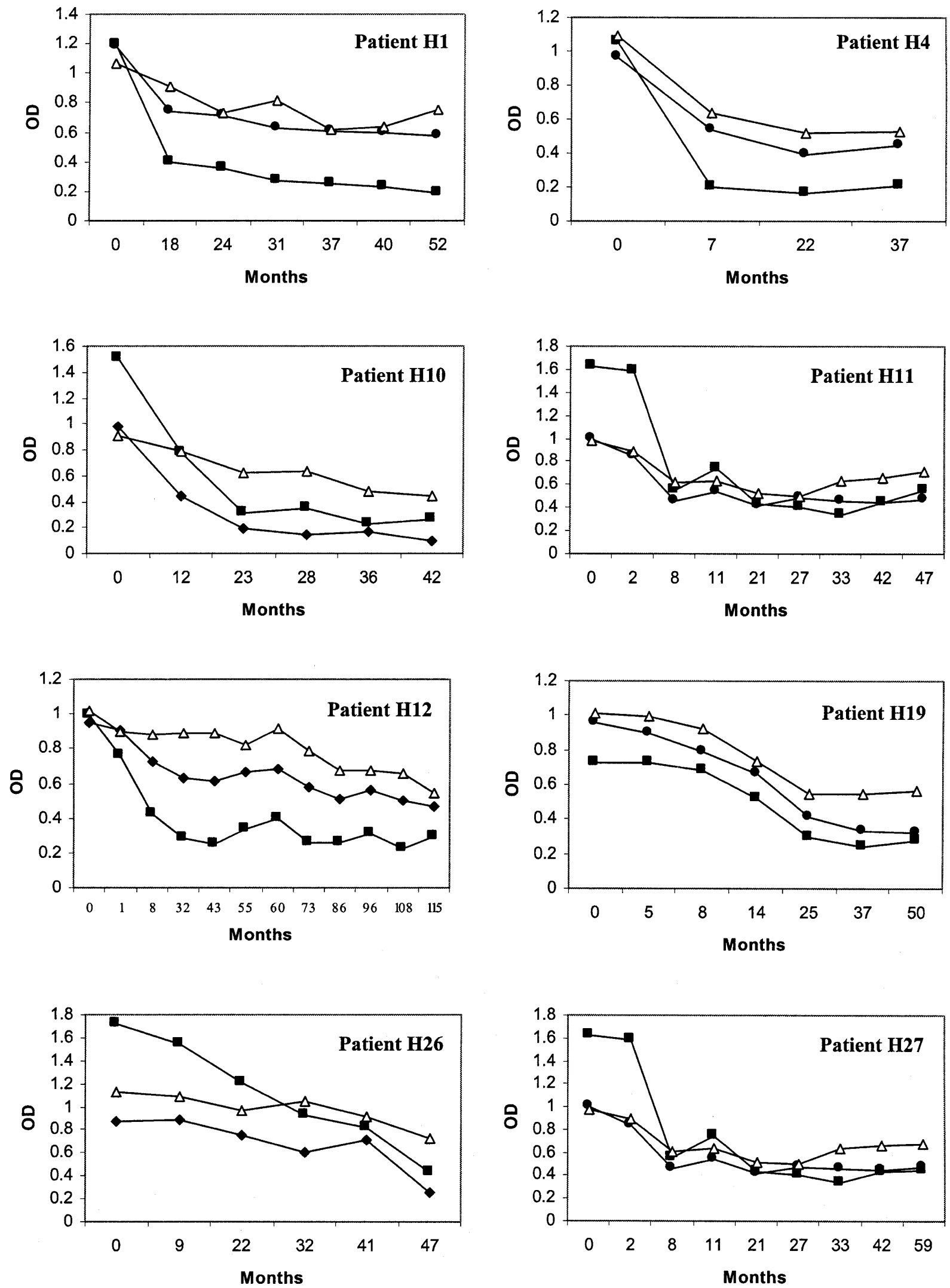

Figure 2. Changes in serum concentrations of total $\operatorname{IgG}(\triangle), \operatorname{IgG1}(\bullet)$, and $\operatorname{IgG} 2(\boldsymbol{\square})$ antibodies among patients $(\mathrm{n}=8)$ in whom cystic echinococcosis (CE) was cured by surgery and anthelminthic chemotherapy but in whom the CE-specific total IgG enzyme-linked immunosorbent assay (ELISA) optical density (OD) values remained $\geq 0.5$ during follow-up. The ELISA ODs are plotted against duration of follow-up with time 0 representing the time of diagnosis and initiation of treatment. 
TABLE 2

Mean \pm SD ELISA OD values of Echinococcus-specific total $\mathrm{IgG}$ and $\mathrm{IgG}$ subclasses in patients $(\mathrm{n}=11)$ at diagnosis of active cystic echinococcosis and during inactive disease*

\begin{tabular}{lccccc}
\hline & Peak OD & Inactive OD & $\Delta$ OD $\dagger$ & No. $\Delta$ OD $\geq 0.2$ & 7 \\
No. $\Delta$ OD $\geq 0.5$ \\
\hline Total IgG & $0.925 \pm 0.284$ & $0.658 \pm 0.318$ & $0.267 \pm 0.191$ & 7 & 1 \\
IgG1 & $0.703 \pm 0.403$ & $0.406 \pm 0.390$ & $0.298 \pm 0.214$ & 9 & 3 \\
IgG2 & $1.318 \pm 0.482$ & $0.799 \pm 0.466$ & $0.520 \pm 0.327$ & 3 & 5 \\
IgG3 & $0.494 \pm 0.707$ & $0.370 \pm 0.495$ & $0.195 \pm 0.364$ & 6 & 2 \\
IgG4 & $0.616 \pm 0.332$ & $0.290 \pm 0.330$ & $0.297 \pm 0.272$ & 6 & 2 \\
\hline
\end{tabular}

* The mean post-treatment decrease in OD $(\Delta \mathrm{OD})$ and the number of patients with a post-treatment decrease in OD $\geq 0.2$ (No. $\Delta$ OD $\geq 0.2)$ and $\geq 0.5$ (No. $\Delta$ OD $\geq 0.5)$ are given. ELISA

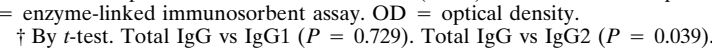

alerted the clinician to the disease activity before it became clinically and radiologically apparent. This information may have led to earlier treatment and thereby limited the morbidity associated with this relapse.

In contrast to our results, previous studies of the correlation of serum concentrations of $\mathrm{IgG}$ subclass antibody responses in patients with symptomatic $\mathrm{CE}$ have indicated that IgG4 antibodies might correlate well with the activity of CE. ${ }^{15,19}$ However, in light of the present findings, it is interesting to note that the $\mathrm{IgG} 2$ antibody response has previously been observed in patients with cysts undergoing natural involution, appearing on ultrasound as a solid mass with or without calcification $^{12}$ (classified as type IV or V cysts, respectively). ${ }^{4}$ Several factors may underlie the differences between our data and those of previous studies. First, previous studies have been limited by a cross-sectional design, whereas in the present study the detailed clinical and radiologic follow-up of our cohort of patients with $\mathrm{CE}$ over many years
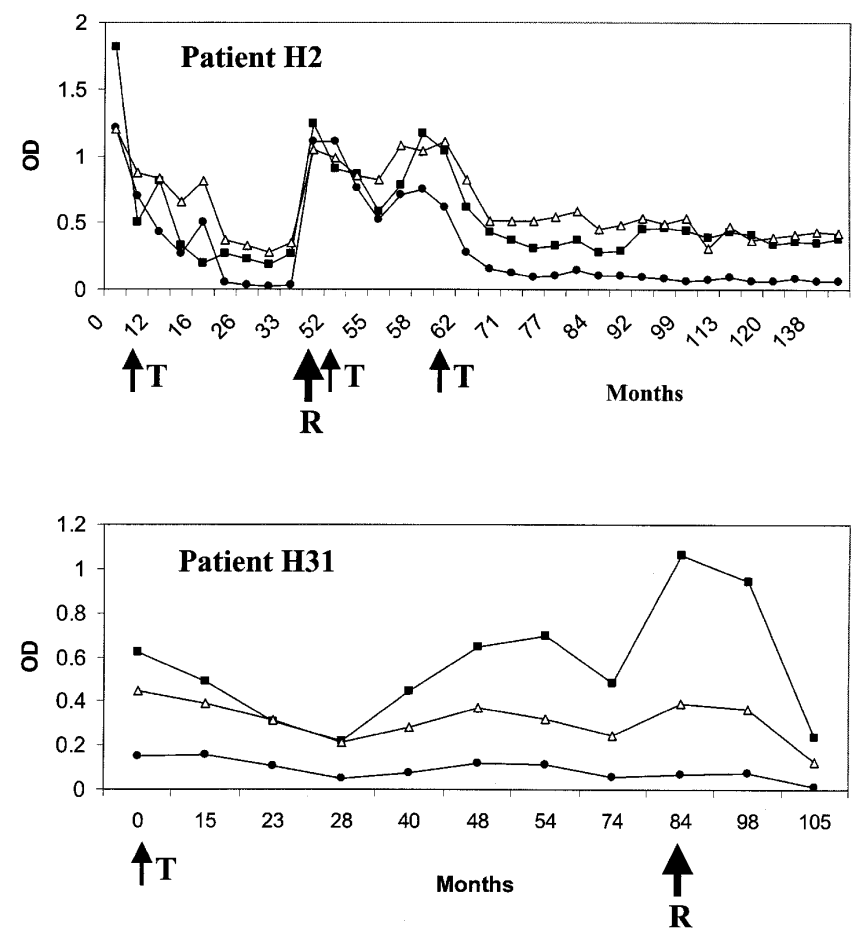

Figure 3. Changes in total $\operatorname{IgG}(\triangle), \operatorname{IgG} 1(\bullet)$, and $\operatorname{IgG} 2(\boldsymbol{\square})$ enzyme-linked immunosorbent assay optical density (OD) values in two patients in whom relapse of cystic echinococcosis (CE) was detected during follow-up. The times at which treatment was given (T) and the time at which a disease relapse occurred $(\mathbf{R})$ are indicated. permitted longitudinal analysis of changes in $\mathrm{IgG}$ subclass antibody responses over time. Second, we used crude horse hydatid cyst antigen, whereas recent studies have most commonly used partially purified hydatid cyst fluid antigen $\mathrm{B},{ }^{14,15,19}$ which appears to preferentially bind $\mathrm{IgG} 4$ antibodies. ${ }^{14,21}$ Indeed, the lack of standardized assays in serologic studies of CE make it difficult to make comparisons between different laboratories. Standardization would greatly facilitate progress of research in this field. Furthermore, the development of the World Health Organization standardized radiologic classification of cysts ${ }^{4}$ will further help comparability of studies.

Since our assays used crude hydatid cyst fluid as antigen, we proceeded to determine which was the immunodominant antigen recognized by the $\mathrm{IgG} 2$ subclass. The lipoprotein antigen $\mathrm{B}$ and antigen 5 are the two major immunodominant antigens that have been identified in E. granulosus cysts and are the most widely used antigens in current assays for immunodiagnosis of CE. ${ }^{22}$ Antigen $\mathrm{B}$ is a polymeric lipoprotein with a molecular mass of $120 \mathrm{kD}$ that dissociates into 8-, 16-, and $24-\mathrm{kD}$ subunits under reducing conditions on immunoblots, whereas antigen 5 is a very high molecular mass lipoprotein complex that dissociates into 38 and $22-24-\mathrm{kD}$ subunits. We found that IgG2 predominantly bound to the $8-\mathrm{kD}$ subunit of antigen B. Thus, the difference between our results and previous data is not simply explained by use of crude cyst fluid as antigen instead of partially purified antigen $\mathrm{B}$.

In conclusion, using ELISAs that incorporated crude $E$. granulosus horse hydatid cyst fluid antigen and CE-specific total $\mathrm{IgG}, \mathrm{IgG} 1$, and IgG2 were found to be sensitive correlates of disease at the time of clinical presentation. However, the IgG2 antibody response was the best correlate of disease activity during post-treatment follow-up. Further prospective evaluation of the $\operatorname{IgG} 2$ antibody response in patients during treatment is warranted using standardized serologic and radiologic reporting methods.

Received October 24, 2003. Accepted for publication December 1, 2003.

Acknowledgment: We are grateful for the administrative assistance provided by Margaret Armstrong.

Authors' addresses: Stephen D. Lawn, Department of Infectious Diseases, St. George's Hospital Medical School, London SW17 ORE, United Kingdom, Telephone: 44-208-725-5828, Fax: 44-208-725-3487, E-mail: stevelawn@yahoo.co.uk. John Bligh, Hospital for Tropical Diseases, London WC1E 6AU, United Kingdom. Philip S. Craig, Cestode Zoonoses Research Group, School of Environment and Life Sciences, University of Salford, Salford M5 4WT, United Kingdom. Peter L. Chiodini, Hospital for Tropical Diseases, London, United Kingdom and Department of Infectious and Tropical Diseases, Lon- 
don School of Hygiene and Tropical Medicine, London WC1E 7HT, United Kingdom.

\section{REFERENCES}

1. Schantz PM, 1991. Parasitic zoonoses in perspective. Int J Parasitol 321: 161-170.

2. Gottstein B, Reichen J, 2003. Echinococcosis/hydatidosis. Cook GC, Zumla A, eds. Manson's Tropical Diseases. London: W. B. Saunders, Elsevier Science Ltd., 1561-1582.

3. Babba H, Messedi A, Masmoudi S, Zribi M, Grillot R, AmbrioseThomas P, Beyrouti I, Sahnoun Y, 1994. Diagnosis of human hydatidosis: comparison between imagery and six serologic techniques. Am J Trop Med Hyg 50: 64-68.

4. WHO/OIE, 2001. Manual on Echinococcosis in Humans and Animals: A Public Health Problem of Global Concern. Eckert J, Gemmell MA, Meslin FX, Pawlowski ZS, eds. Geneva: World Health Organization.

5. Richards KS, Morris DL, 1990. Effect of albendazole on human hydatid cysts: an ultrastructural study. HPB Surg. 2: 105-112.

6. Moskopp D, Lotterer E, 1993. Concentrations of albendazole in serum, cerebrospinal fluid and hydatidous brain cyst. Neurosurg Rev 16: 35-37.

7. Morris DL, Richards KS, Chinnery JB, 1986. Protoscolicidal effect of praziquantel-in-vitro and electron microscopical studies on Echinococcus granulosus. J Antimicrob Chemother 18: 687-691.

8. Taylor DH, Morris DL, 1989. Combination chemotherapy is more effective in postspillage prophylaxis for hydatid disease than either albendazole or praziquantel alone. Br J Surg 76: 954.

9. Boctor FN, Peter JB, 1990. IgG subclasses in human chronic schistosomiasis: over-production of schistosome-specific and non-specific IgG4. Clin Exp Immunol 82: 574-578.

10. Short JA, Heiner DC, Hsiao RL, Andersen FL, 1990. Immunoglobulin E and G4 antibodies in cysticercosis. J Clin Microbiol 28: $1635-1639$.

11. Kurniawan A, Yazdanbakhsh M, van Ree R, Aalberse R, Selkirk ME, Partono F, Maizels RM, 1993. Differential expression of $\mathrm{IgE}$ and $\mathrm{IgG} 4$ specific antibody responses in asymptomatic and chronic human filariasis. J Immunol 150: 3941-3950.
12. Daeki AO, Craig PS, Shambesh MK, 2000. IgG-subclass antibody responses and the natural history of hepatic cystic echinococcosis in asymptomatic patients. Ann Trop Med Parasitol 94: 319-328.

13. Aceti A, Pennica A, Teggi A, Fondacaro LM, Caferro M, Leri O, Tacchi G, Celestino D, Quaranta G, de Rosa F, Sebastiani A, 1993. IgG subclasses in human hydatid disease: prominence of the IgG4 response. Int Arch Allergy Immunol 102: 347-351.

14. Wen H, Craig PS, 1994. Immunoglobulin G subclass responses in human cystic and alveolar echinococcosis. Am J Trop Med Hyg 51: 741-748.

15. Shambesh MK, Craig PS, Wen H, Rogan MT, Paolillo E, 1997. $\mathrm{IgG1}$ and IgG4 serum antibody responses in asymptomatic and clinically expressed cystic echinococcosis patients. Acta Trop 64: 53-63.

16. Ayles HM, Corbett EL, Taylor I, Cowie AG, Bligh J, Walmsley $\mathrm{K}$, Bryceson AD, 2002. A combined medical and surgical approach to hydatid disease: 12 years' experience at the Hospital for Tropical Diseases, London. Ann R Coll Surg Engl 84: 100105.

17. Filice C, Brunetti E, 1997. Use of PAIR in human cystic echinococcosis. Acta Trop 64: 95-107.

18. Harlow E, Lane D, 1988. Immunoblotting. Antibodies: A Laboratory Manual. Cold Spring Harbor, NY: Cold Spring Harbor Laboratory Press, 471-509.

19. Rigano R, Profumo E, Ioppolo S, Notargiacomo S, Ortona E, Teggi A, Siracusano A, 1995. Immunological markers indicating the effectiveness of pharmacological treatment in human hydatid disease. Clin Exp Immunol 102: 281-285.

20. Ramzy RM, Helmy H, El Zayyat EA, Rifaat MM, Abdel Hameed DM, Abdel-Baki MH, 1999. An enzyme-linked immunosorbent assay for detection of IgG1 antibodies specific to human cystic echinococcosis in Egypt. Trop Med Int Health 4: 616-620.

21. McVie A, Ersfeld K, Rogan MT, Craig PS, 1997. Expression and immunological characterisation of Echinococcus granulosus recombinant antigen B for IgG4 subclass detection in human cystic echinococcosis. Acta Trop 67: 19-35.

22. Zhang W, Li J, McManus DP, 2003. Concepts in immunology and diagnosis of hydatid disease. Clin Microbiol Rev 16: 18-36. 